\title{
Electrical Stimulation of the Horizontal Limb of the Diagonal Band of Broca Modulates Population EPSPs in Piriform Cortex
}

\author{
CHRISTIANE LINSTER, BRADLEY P. WYBLE, AND MICHAEL E. HASSELMO \\ Department of Psychology and Program in Neuroscience, Harvard University, Cambridge, Massachusetts 02138
}

\begin{abstract}
Linster, Christiane, Bradley P. Wyble, and Michael E. Hasselmo. Electrical stimulation of the horizontal limb of the diagonal band of Broca modulates population EPSPs in piriform cortex. $J$. Neurophysiol. 81: 2737-2742, 1999. Electrical stimulation of the horizontal limb of the diagonal band of Broca (HDB) was coupled with recording of evoked potentials in the piriform cortex. Stimulation of the HDB caused an enhancement of the late, disynaptic component of the evoked potential elicited by stimulation of the lateral olfactory tract but caused a suppression of the synaptic potential elicited by stimulation of the posterior piriform cortex. The muscarinic antagonist scopolamine blocked both effects of HDB stimulation. The enhancement of disynaptic potentials could be due to cholinergic depolarization of pyramidal cells, whereas the suppression of potentials evoked by posterior piriform stimulation could be due to presynaptic inhibition of intrinsic fiber synaptic transmission by acetylcholine.
\end{abstract}

\section{N T R O D U C T I O N}

Modulatory effects of acetylcholine have been shown to play a role in olfactory behavior. In particular, the muscarinic antagonist scopolamine has been shown to impair short-term memory for odors (Ravel et al. 1994), habituation to familiar odors (Hunter and Murray 1989), and odor-based social recognition (Perio et al. 1989; Soffie and Lambert 1988). Lesions of the cholinergic and GABAergic inputs from the basal forebrain have also been shown to influence olfactory habituation and investigation (Paolini and McKenzie 1993, 1996).

Computational models of the olfactory system have investigated how the specific effects of cholinergic modulation on neuronal circuits could influence processing of olfactory stimuli (Hasselmo 1993; Linster and Gervais 1996; Linster and Hasselmo 1997a). The implementation of cholinergic effects in the piriform cortex in these models has been based primarily on experimental data from brain slice preparations. These data include experiments showing that cholinergic agonists depolarize piriform cortex pyramidal cells (Barkai and Hasselmo 1994), suppress neuronal adaptation (Barkai and Hasselmo 1994; Tseng and Haberly 1989), and suppress excitatory and inhibitory synaptic transmission in the association fiber pathway (Hasselmo and Bower 1992; Patil and Hasselmo 1996; Williams and Constanti 1988a).

Although brain slice preparations provide controlled conditions for analyzing modulatory effects, it is important to demonstrate that these effects appear in in vivo preparations. This ensures that the effect appears with neuronal release of the modulator in anatomically intact circuits. Both the olfactory

The costs of publication of this article were defrayed in part by the payment of page charges. The article must therefore be hereby marked "advertisement" in accordance with 18 U.S.C. Section 1734 solely to indicate this fact. bulb (OB) and piriform cortex receive cholinergic input from the horizontal limb of the diagonal band of Broca (HDB) (Zaborsky et al. 1986a). The neurons in the HDB contain both ACh and GABA (Brashear et al. 1986), and it is known that the cholinergic and GABAergic neurons in the HDB are at least partially spatially segregated (Brashear et al. 1986). This spatial segregation of cholinergic and GABAergic neurons in different regions of the HDB allows for preferential stimulation of cholinergic neurons projecting to the $\mathrm{OB}$ and/or piriform cortex (Luskin and Price 1982; Zaborszky et al. 1986b). Several studies of cholinergic effects in the OB have been performed in vivo (Elaagouby et al. 1991; Kunze et al. 1991, 1992; Nickell and Shipley 1988, 1993; Ravel et al. 1990), but to our knowledge only a single study has investigated the effects of cholinergic modulation in the piriform cortex in vivo (Zimmer et al. 1996).

We investigate the effect of electrical stimulation in the HDB on excitatory synaptic transmission in the piriform cortex in vivo. We investigate the effect of HDB stimulation on the population excitatory postsynaptic potentials (EPSPs) evoked by stimulation of the lateral olfactory tract (LOT) and more posterior piriform cortex (pPC). We observe 1 ) no effect on the monosynaptic EPSP evoked by electrical stimulation of the LOT, 2) an increase of the disynaptic component of the population EPSP evoked by LOT stimulation, and 3) a decrease of the population EPSP evoked by electrical stimulation of intrinsic association fibers from the pPC. All the observed effects are significantly decreased under the muscarinic antagonist scopolamine, suggesting that they are at least partially mediated by cholinergic pathways.

\section{E T H O D S}

\section{Surgical preparation}

Adult, male Sprague Dawley rats (300-400 g) were anesthetized with urethan $(1.5 \mathrm{~g} / \mathrm{kg}$ ip). Levels of anesthesia were monitored by rate of respiration and foot withdrawal reflex and supplemented if necessary by intraperitoneal injections. Body temperature was maintained at $37^{\circ} \mathrm{C}$ with a heating pad. Anesthetized animals were placed in a stereotaxic apparatus, and the skull was exposed by scalp incision. We used a total of 11 animals for the data presented here. In seven animals, both stimulation of the LOT and stimulation of the pPC were used. Holes were drilled in the skull at positions appropriate for gaining access to the stereotaxic locations (Paxino and Watson 1986) of the LOT, the HDB, anterior piriform cortex (aPC), and pPC. Recently, it has been shown that urethan has an effect on population EPSPs such as those observed in the experiments described here (Cauthron and Stripling 1998); however, the effects measured in our experiments (relative size of evoked potentials with and without 
electrical stimulation of cholinergic afferents) should not be affected by the tonic presence of urethan anesthesia.

\section{Electrical stimulation}

As summarized schematically in Fig. 2, bipolar stimulation electrodes $(100 \mu \mathrm{m}$ stainless steel, Formvar-insulated except at the tips, twisted together) were placed in the LOT $(4.7 \mathrm{~mm}$ anterior; $3.4 \mathrm{~mm}$ lateral; 5.2 ventral from bregma), in $\mathrm{pPC}(3.6 \mathrm{~mm}$ posterior, $3.0 \mathrm{~mm}$ lateral ( $14^{\circ}$ angled laterally); $9-10 \mathrm{~mm}$ ventral from bregma), and in the HDB $(0.0-0.5 \mathrm{~mm}$ posterior, $1.8-2.2 \mathrm{~mm}$ lateral, $8.6-9.0 \mathrm{~mm}$ ventral from bregma). We chose the coordinates for the HDB stimulation electrode in such a way as to ensure a high probability for stimulation of cholinergic neurons projecting to the olfactory system, based on anatomic data (Brashear et al. 1986; Luskin and Price 1982; Zaborszky et al. 1986b). Optimal placement of the recording electrode was achieved by monitoring the field potentials evoked in layer Ia of aPC. All stimulation currents were delivered by a constant current stimulus isolation unit (Grass Instruments) controlled by a Grass Instrument stimulator. Stimulation currents for the LOT and pPC electrodes were adjusted to approximately two times threshold ([SIM]0.3 ms pulses at 100-300 $\mu \mathrm{A}$ ). After placement, stimulation strength for the HDB electrode was adjusted such as to evoke no field potential response in aPC $(\sim 100-300 \mu \mathrm{A})$. For placement of the pPC electrode, we recorded the field potentials evoked by LOT stimulation from this electrode and placed the electrode in the deeper layers (II or III) of pPC, as determined by the polarity of the response observed in response to LOT stimulation (Haberly 1973). Field potentials elicited by stimulation of layer I of the piriform cortex can easily be contaminated by antidromic LOT activation, and the components of these field potentials are difficult to interpret. We therefore chose to stimulate layer II-III of pPC, evoking a population EPSP in aPC, presumably caused by direct electrical activation of the deeper layer association fibers. This produced a positive-going potential in layer Ia of aPC, which reversed near layer II. The locations of stimulus electrodes and the recording electrode are summarized schematically in Fig. 1.

\section{Recording}

For recording of field potentials, a recording electrode $(100 \mu \mathrm{m}$ stainless steel, formvar insulated except at the tip) was placed in layer Ia of the aPC in such a way as to observe a short latency response to stimulation of the LOT. DC preamplification (Grass Instruments, $\times 10$ ) was used in all recordings; the signal was then further amplified $(\times 200)$ and filtered between 0.1 and $6000 \mathrm{~Hz}$ via a Neuralynx differential amplifier. Data acquisition, display, and control of stimulus were done by use of computer (Pentium $100 \mathrm{MHz}$ and Datawave Workbench 32 software). Amplitude of field potentials was measured from the baseline ( $0-2 \mathrm{~ms}$ before the stimulus artifact) to the peak of the negative deflection (when stimulating LOT) or the positive deflection (when stimulating layer II-III of pPC).

\section{Drug administration}

Systemic injections of scopolamine $(0.5 \mathrm{mg} / \mathrm{kg}$ ip in saline) were used to test whether the observed effects were due to activation of muscarinic cholinergic receptors. The difference between the baseline pulses and pulses after HDB stimulation was compared before and after the scopolamine injections.

\section{Histology}

At the end of the experiment, current was passed through the recording and stimulation electrodes (via a $9-\mathrm{V}$ battery) to deposit iron in the tissue. Transcardial perfusion was then performed with saline and potassium ferrocyanide in $10 \%$ buffered formalin. After storage in
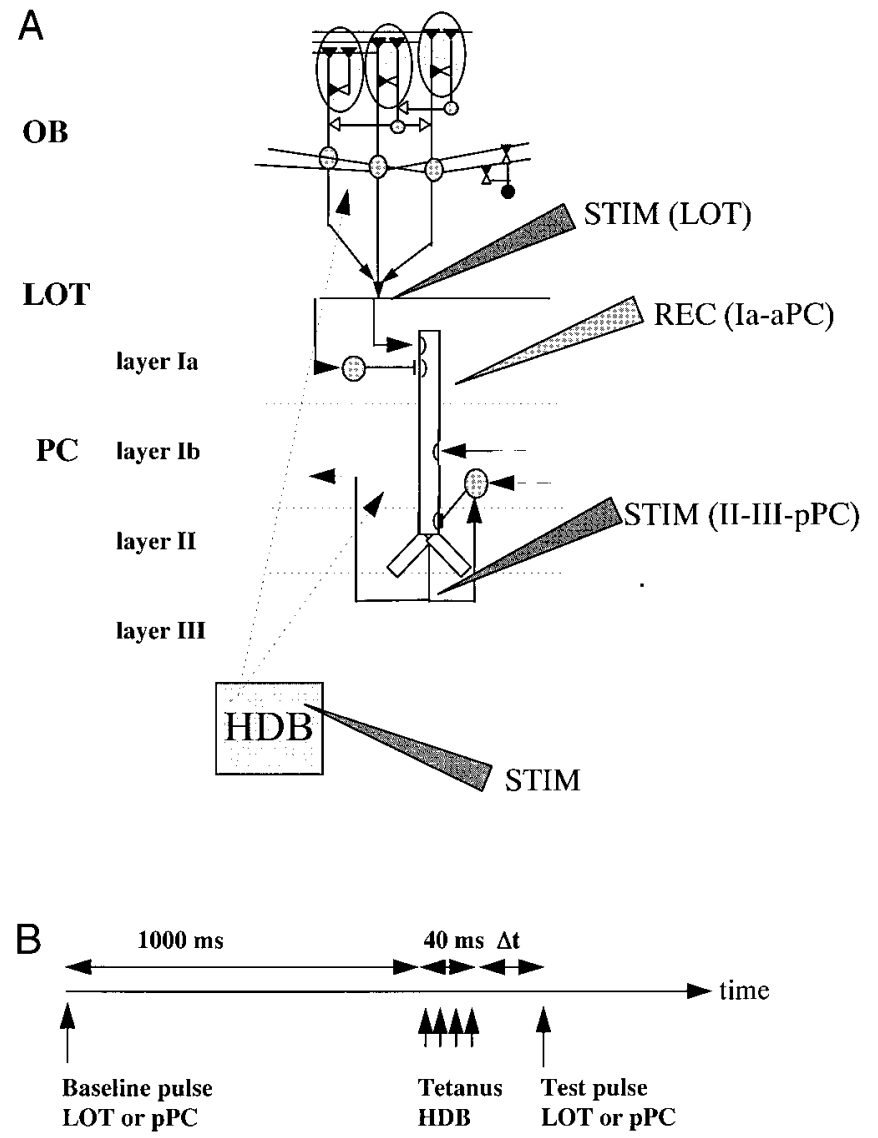

FIG. 1. Experimental protocol. A: schematic diagram of the experimental setup. Stimulation electrodes (STIM) were positioned in the lateral olfactory tract (LOT), horizontal limb of the diagonal band (HDB), and deeper layers (II-III) of posterior piriform cortex (PC). A recording electrode (REC) was positioned in layer Ia of anterior piriform cortex (aPC). OB: olfactory bulb; PC: piriform cortex. $B$ : each experiment consisted of a set of 10 trials repeated at 1-min intervals. A baseline pulse (LOT or pPC) was given, and the response to this baseline pulse was recorded. After $1,000 \mathrm{~ms}, 4$ consecutive pulses at 100 $\mathrm{Hz}$ (tetanus HDB) are delivered through the stimulation electrode in the HDB. The response to a second pulse (test pulse) similar to the baseline pulse (LOT or $\mathrm{pPC}$ ) was then recorded after a variable delay $\Delta t$. In the experiments reported here, $\Delta t$ was chosen to be $25,50,100$, and $250 \mathrm{~ms}$. For each animal, the ratios of the response amplitudes between the test pulse and the baseline pulse was calculated. In a control experiment, the stimulation current in the HDB electrode was very low $(<10 \mu \mathrm{A})$. The ratios of the responses in the control experiment served as baseline for the statistical tests ( $t$-test, single tailed, significance if $P<0.01$ ).

$10 \%$ formalin with $20 \%$ sucrose, brains were sectioned and stained with Neutral red for localization of the recording and stimulating electrodes.

\section{Experimental protocol}

These experiments quantified the effect of electrical stimulation of the HDB on evoked responses in aPC (Fig. 1). As reported previously by other authors for similar experimental protocols (Nickell and Shipley 1993; Zimmer et al. 1996), we could not observe an effect when single electrical pulses were used for stimulation in the HDB. We therefore tested the effect of a brief tetanus ( 4 pulses of $0.3-\mathrm{ms}$ width separated by $10 \mathrm{~ms}$ ) in the HDB on the evoked responses to a subsequent single $0.3-\mathrm{ms}$ pulse in either the LOT or the pPC. A single baseline pulse was given in the LOT or pPC electrode, and the response in the aPC was recorded (baseline pulse). After a delay of $1 \mathrm{~s}$, the tetanus in the HDB electrode was given followed by a second 

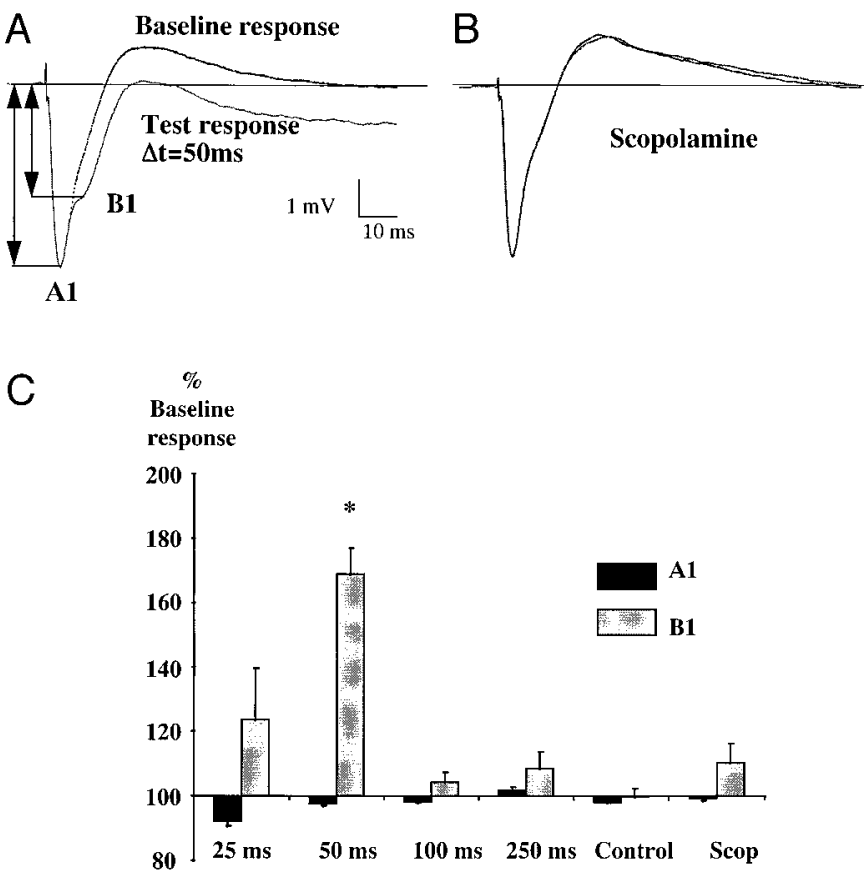

FIG. 2. Effect of stimulation of HDB on the population EPSP in layer Ia of piriform cortex in response to stimulation of the lateral olfactory tract. $A$ : responses to baseline pulse (baseline response) and test pulse $50 \mathrm{~ms}$ after HDB stimulation (test response, $\Delta t=50 \mathrm{~ms}$ ). The population EPSP observed in layer Ia of the PC after stimulation of the LOT has a first negative peak (A1), followed by a second negative inflection (B1). A1 is generated by the monosynaptic excitatory postsynaptic potential (EPSP) in layer Ia, and B1 is thought to reflect the disynaptic EPSP caused by activation of the intrinsic fibers within piriform cortex; $50 \mathrm{~ms}$ after the tetanus in the $\mathrm{HDB}$, component $\mathrm{B} 1$ is greatly enhanced. There is no effect on the monosynaptic component A1. Each trace is the average of 10 stimulations. The lines with arrows to the left of the potential indicate the measurements of the amplitude of the A1 and B1 components used for the analysis. $B$ : responses to baseline pulse and test pulse $50 \mathrm{~ms}$ after HDB stimulation $30 \mathrm{~min}$ after the injection of $0.5 \mathrm{mg} / \mathrm{kg}$ scopolamine. Scopolamine abolishes or greatly reduces the enhancement of component B1 after HDB stimulation. Each trace is the average of 10 stimulations. $A$ and $B$ are from the same animal. $C$ : average results (shown as average \pm SE) obtained in 8 animals. Results are shown as percentages of the response to the baseline pulse (100\%); $50 \mathrm{~ms}$ : a maximal effect on component B1 was observed when the LOT was stimulated $50 \mathrm{~ms}$ after the HDB tetanus. Control: the same stimulation protocol was used $(\Delta t=50 \mathrm{~ms})$ but the stimulation current in the HDB is $<10 \mu \mathrm{A}$. Scop: response measured with a 50 -ms delay $30 \mathrm{~min}$ after injection of $0.5 \mathrm{mg} / \mathrm{kg}$ scopolamine (in 6 animals) with the same stimulation currents that previously caused enhancement $(\Delta t=$ $50 \mathrm{~ms}$ ). The effect on component B1 observed at $50 \mathrm{~ms}$ was highly significant $(P<0.01)$ when compared with the control experiment; no significant effect was obtained at other delays $\Delta t$ or in the scopolamine condition. Significant effects are indicated by *.

single test pulse in the LOT or pPC electrode (test pulse). The delay $(\Delta t)$ between the HDB tetanus and the LOT or pPC test pulse was variable $(\Delta t=25,50,100$, and $250 \mathrm{~ms})$. The stimulation current in the HDB electrode was adjusted to be just below threshold for the observation of a population response in the aPC. In the control experiment, the HDB stimulation was given with very low currents $(<10 \mu \mathrm{A})$ at $\Delta t=50 \mathrm{~ms}$. For each delay $\Delta t$ and for the control, a set of 10 trials was performed with a 1-min interval between each trial. For each trial, the response to the test pulse after HDB stimulation was compared with the response to the baseline pulse. For the monosynaptic component of the population EPSPS, we measured both the amplitude of the peak and the slope of the rising phase of the potentials (measured between baseline 1-2 ms before the stimulation artifact and the peak response). For the disynaptic phase of the LOT-evoked response (B1), an accurate measurement of the slope cannot be obtained; therefore only amplitude measurements were obtained. The amplitude of the B1 response was quantified by using a time point determined by visual inspection and measuring the distance from the prestimulus baseline to the physiological trace at that time point (see Fig. 2). The point chosen by visual inspection corresponded as accurately as possible to the minimum absolute value of the first derivative of the trace (i.e., the point where the rate change was smallest). This was done on the basis of the trace with the clearest $\mathrm{B} 1$ response (usually the test response) for each experiment. In cases were a peak was clearly visible, the time point of the local minimum was chosen. The point of measurement of B1 was usually $\sim 8 \mathrm{~ms}$ after the first peak (A1). The results obtained from amplitude and slope measurements were similar in all cases. The results are reported as the ratio between the rising slope (or the amplitude) of the response to the test pulse after HDB stimulation and the response to the baseline pulse. For each delay $\Delta t$, this ratio is then compared with the ratio obtained in the control condition (low stimulation current in HDB electrode). The experiments (same electrode locations and stimulation currents) were repeated $30 \mathrm{~min}$ after injection of $0.5 \mathrm{mg} / \mathrm{kg}$ ip scopolamine. Results are reported as average \pm SE. Statistical tests ( $t$-test, single-tailed) were performed to determine significance $(P<$ 0.01 ) of results.

\section{RES U L T S}

\section{Effect of HDB stimulation on the population EPSP evoked by stimulation of the LOT}

The population EPSP observed in layer Ia of the PC by stimulation of the LOT (Fig. $2 A$ ) contains a number of different components that have been described in detail (Haberly 1973; Ketchum and Haberly 1993). The first negative peak has been labeled period 1 and is composed of two phases called A1 and B1. A1 is generated by the monosynaptic EPSP in layer Ia, and B1 has been proposed to result from a disynaptic EPSP caused by activation of recurrent collaterals within the piriform cortex. The following positive component is called period 2 and is thought to result from inhibitory input to pyramidal cells. In the experiment described here, we observed the effect of a brief tetanus in the HDB on period 1 of the population EPSP evoked by LOT stimulation.

No significant effect of HDB stimulation on the monosynaptic population EPSP (A1) was observed in our experiments ( $n=8$; Fig. 2), as previously reported by Zimmer et al. (1996). In contrast, component B1 of the population EPSP was increased by the preceding stimulation in the HDB (Fig. 2B). As shown in Fig. $2 C$, a significant effect $(169 \pm 7.3 \%$ of the baseline response; $n=8 ; P<0.01$ ) was observed, only for $\Delta t=50 \mathrm{~ms}$. This stimulation induced increase in B1 was blocked by scopolamine; $30 \mathrm{~min}$ after injection of $0.5 \mathrm{mg} / \mathrm{kg}$ scopolamine, no significant effect was observed after the tetanus in the HDB electrode with a delay of $\Delta t=50 \mathrm{~ms}(110 \pm$ $6 \%$ of test response; $n=6 ; P>0.2$ ). Scopolamine had no effect on the response to the test pulse in the LOT. Thus the baseline used for comparison did not change. Figure 2 summarizes the effects on the population EPSP evoked by stimulation of the LOT.

\section{Modulation of the population EPSP evoked by stimulation of the $p P C$}

For these measurements, the stimulation electrode was placed into layers II-III of pPC. Electrical stimulation of the deeper layers of pPC evokes a complex population response 

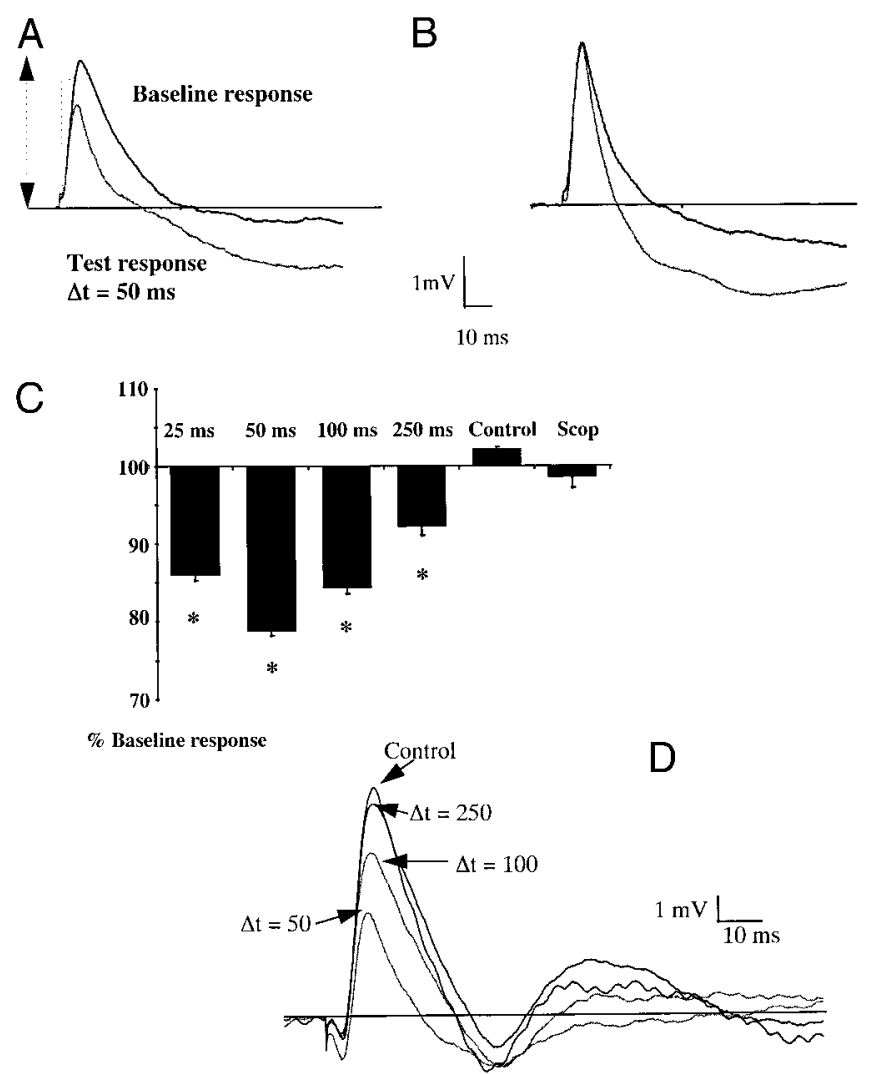

FIG. 3. Effect of stimulation of HDB on the population EPSP in layer Ia in response to stimulation of layers II-III in posterior piriform cortex. A: responses to baseline pulse (baseline response) and test pulse $50 \mathrm{~ms}$ after HDB stimulation $(\Delta t=50 \mathrm{~ms})$. In these experiments, we considered only the first peak of the response, which represents the monosynaptic population EPSP and which could be reliable obtained at a short latency. The line with arrow indicates the measurement of the peak of the response used in the analysis; the pointed lines show the measurement of the onset slope. After stimulation of the HDB, the first positive peak was reduced in most animals. $B$ : responses to baseline pulse and test pulse $50 \mathrm{~ms}$ after HDB stimulation $30 \mathrm{~min}$ after the injection of $0.5 \mathrm{mg} / \mathrm{kg}$ scopolamine. Scopolamine abolished or greatly reduced the suppression of the first peak after HDB stimulation. Each trace is the average of 10 stimulations. $A$ and $B$ are from the same animal. $C$ : average results (shown as average $\pm \mathrm{SE}$ ) obtained in 10 animals. Results are show as percentages of the response to the test pulse $(100 \%)$. The average suppression obtained at different delays $\Delta t$ are shown. The maximal effect is obtained for $\Delta t=50 \mathrm{~ms}$, but a significant effect could still be seen at 100 and $250 \mathrm{~ms}$. Control: the same stimulation protocol was used $(\Delta t=50 \mathrm{~ms})$, but the stimulation current in the HDB is $<10 \mu \mathrm{A}$. Scop: response measured with a 50 -ms delay $30 \mathrm{~min}$ after injection of $0.5 \mathrm{mg} / \mathrm{kg}$ scopolamine $(n=8)$. The effects observed with delays of 50 and $100 \mathrm{~ms}$ were highly significant $(P<$ 0.01 ) when compared with the control experiment; no significant effect was obtained at either delay in the scopolamine condition. $D$ : comparison of responses recorded at different intervals $\Delta t$ after the HDB stimulation and the test response. Traces are averages of 10 responses from the same experiment.

recorded in layer Ia of aPC, of which we considered only the first peak, which is presumably the monosynaptic response evoked by stimulation of intrinsic association fibers and which could be reliably evoked in our experiments. When recorded in layer Ia of aPC, the first deflection of the response evoked by stimulation of the deeper layers is positive. Consistent with anatomic data, this potential reverses from positive to negative around the same location as the response evoked by stimulation of the LOT reverses from negative to positive. The first peak is negative if recorded in the deeper layers (layers II-III) of aPC, suggesting that we are stimulating association fibers that ter- minate in layers II-III or in the most proximal portion of layer Ib (Haberly 1985). In most animals tested (8/10), a significant decrease in the population EPSP evoked by stimulation of the deeper layers of $\mathrm{pPC}$ was observed after a tetanus in the HDB (Fig. 3A). The maximal decrease was observed when the pPC pulse occurred $50 \mathrm{~ms}$ after the HDB stimulation $(78 \% \pm 0.6 \%$ of the test response; $P<0.0001, n=10$ ) (Fig. 3, $A$ and $C$ ). In contrast to the effect observed for LOT stimulation, the effect on the pPC-evoked EPSP was still significant at a delay of 100 $\mathrm{ms}(84 \% \pm 0.8 \%$ of test response) and at a delay of $250 \mathrm{~ms}$ $(92 \% \pm 1.2 \%$ of test response). Injection of $0.5 \mathrm{mg} / \mathrm{kg} \mathrm{sco-}$ polamine completely abolished the observed decrease in size induced by HDB stimulation $(n=8)$. Interestingly, scopolamine by itself had an effect on the amplitude of the test pulse. When the deeper layers were stimulated, $30 \mathrm{~min}$ after the administration of $0.5 \mathrm{mg} / \mathrm{kg}$ scopolamine, the average rising slope of the population EPSP increased to $146 \pm 5.2 \%$ of the response before scopolamine injection, suggesting that these potentials are under tonic suppression by muscarinic receptor activation.
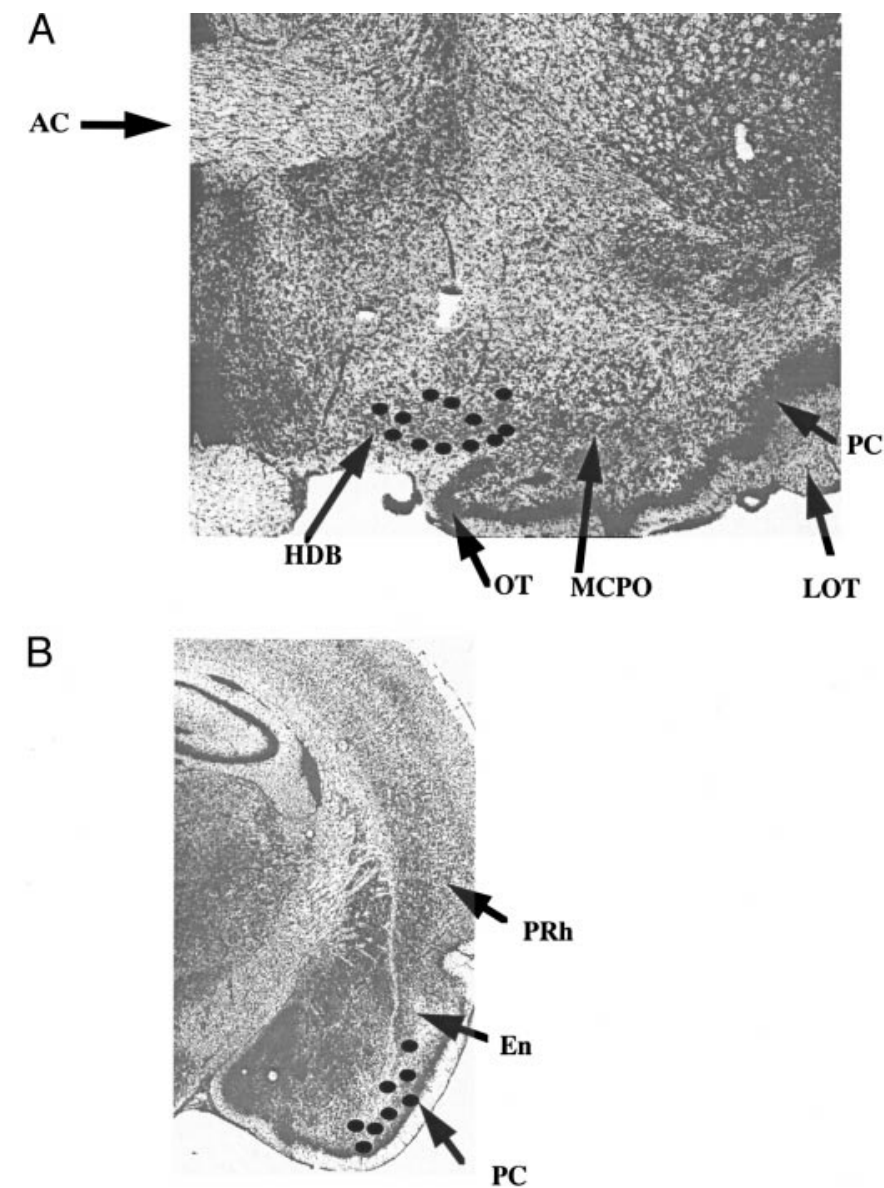

FIG. 4. Location of stimulation electrodes in HDB and in posterior piriform cortex. A: schematic representation of the location of stimulation electrodes in the HDB. All recording sites were located in sections between 0.26 anterior to Bregma and -0.36 posterior to Bregma but are shown here in a single section. HDB: horizontal limb of the diagonal band of Broca; MCPO: magnocellular preoptic nucleus; LOT: lateral olfactory tract; PC: piriform cortex; OT: olfactory tubercle. $B$ : schematic representation of the location of the stimulation electrodes in posterior piriform cortex approximately -3.6 posterior from Bregma. PC: piriform cortex; En: endopiriform nucleus; PRh: Perirhinal cortex. 


\section{Stimulation sites}

After each experiment, all recording and stimulation sites were verified histologically. Only animals in which the stimulation sites were located in the area designated as HDB (Paxino and Watson 1986) were considered for the analysis of the results described here. Figure 4 shows a summary of the lesion sites produced by the HDB electrode (Fig. 4A) and the pPC electrode (Fig. 4B). The locus of stimulation was in the area of the HDB shown to contain a high density of cholinergic neurons projecting to the olfactory system (Brashear et al. 1986; Luskin and Price 1982; Zaborszky et al. 1986b).

\section{I S C U S S I O N}

The results presented here demonstrate that electrical activation of cholinergic input from the horizontal limb of the diagonal band modulates evoked synaptic potentials in the piriform cortex, causing 1) suppression of monosynaptic potentials evoked by stimulation of $\mathrm{pPC}, 2$ ) enhancement of the disynaptic activity induced by LOT stimulation, and 3) no change in the monosynaptic activity induced by LOT stimulation. These results are consistent with brain slice physiological experiments showing cholinergic suppression of intrinsic synaptic potentials in layer Ib with little or no effect on synaptic potentials elicited by afferent fiber stimulation in layer Ia (Hasselmo and Bower 1992).

The synaptic potentials elicited by stimulation of the pPC probably arise from associative connections from $\mathrm{pPC}$ to $\mathrm{aPC}$, in contrast to the potentials elicited by LOT stimulation, which involve induction of both afferent synaptic currents in layer Ia and associative synaptic currents in layers $\mathrm{Ib}$ and in deeper layers (Biella and DeCurtis 1993; Ketchum and Haberly 1993). Our results suggest that the observed suppression of the population EPSP is mediated by muscarinic receptors, as systemic injections of scopolamine reduce the suppression here in the same manner that scopolamine blocks suppression of potentials induced by carbachol in slice preparations of the piriform cortex (Hasselmo and Bower 1992; Williams and Constanti 1988a,b).

The enhancement of disynaptic activity evoked by LOT stimulation probably results from the direct cholinergic depolarization of piriform cortex neurons described in vitro (Barkai and Hasselmo 1994; Tseng and Haberly 1989), which seems to cause a sufficient increase in spiking of pyramidal cells and disynaptic transmission to overcome the simultaneous suppression of intrinsic synaptic transmission.

\section{Relation to previous in vivo results}

The data presented here complements results from previous work in the OB showing that stimulation of the HDB suppresses synaptic potentials elicited in the OB by stimulation of the anterior commissure (Nickell and Shipley 1993). The commissural connections from one $\mathrm{OB}$ to the granule cells in the contralateral $\mathrm{OB}$ could be considered analogous to intrinsic connections between the $\mathrm{pPC}$ and the aPC, although suppression of transmission at these synapses has not been investigated in slice preparations of the OB. The enhancement of disynaptic potentials elicited by LOT stimulation here could result from an increase in excitability of pyramidal cells; indeed previous results reported in the piriform cortex (Zimmer et al. 1996) show an enhancement of spontaneous activity in piriform cortex pyramidal cells after electrical stimulation of the HDB.

A similar cholinergic modulation of synaptic transmission has been shown in in vivo experiments in the hippocampal formation. Iontophoretic injections of acetylcholine or muscarine into stratum radiatum of region CA1 have been shown to suppress population EPSPs elicited by stimulation of the fimbria (Rovira et al. 1982, 1983). Repetitive stimulation of the medial septum also causes suppression of evoked synaptic potentials in stratum radiatum of region CA1 (Rovira et al. 1983) in an experimental paradigm similar to the experiments presented here. Systemic injections of scopolamine blocked the observed effect in that study as well, suggesting that it is at least partially mediated by muscarinic receptors. Other studies have demonstrated the stimulation of the medial septum causes enhancement of population spikes in the pyramidal cell layer of region CA1 (Ben Ari et al. 1981; Krnjevic and Ropert 1981, 1982) and the granule cell layer of the dentate gyrus (Bilkey and Goddard 1985; Fantie and Goddard 1982). This effect shows a rapid time course similar to the effect described here and could result from cholinergic depolarization of pyramidal cells, as suggested by our results for piriform cortex pyramidal cells.

\section{Functional significance}

Our results support the notion that cholinergic modulation of population EPSPs occurs within the intact animal. We observe an enhancement of the disynaptic potential elicited by LOT stimulation, which may result from depolarization of pyramidal cells. We also observe a suppression of the population EPSP evoked by stimulation of intrinsic association fibers. These two effects are complementary; a depolarization of pyramidal cells accompanied by a suppression of intrinsic association fiber transmission can increase the signal-to-noise ratio in response to afferent input from the LOT (Hasselmo et al. 1997). Previous computational modeling work suggests that this cholinergic modulation might allow afferent input to have a dominant influence on physiological activity in the piriform cortex (Barkai et al. 1994; Hasselmo and Linster 1997; Hasselmo et al. 1992). The results presented here show that during activation of the HDB the influence of intrinsic connections arising from $\mathrm{pPC}$ is considerably weakened relative to the unchanged magnitude of monosynaptic afferent input. A dominant influence of afferent input, enhanced by the depolarization of the pyramidal cells, would be appropriate for learning of new information with minimal interference from previously stored representations (Hasselmo and Bower 1993; Hasselmo and Linster 1997b). This would be particularly appropriate for the proposed function of the piriform cortex as an associative memory network (Haberly 1985; Haberly and Bower 1989) because neural network models of associative memory require greater influence of afferent input during storage of information than during retrieval.

The authors are thankful to J. Goodman, L. Zimmer, and Dr. Celeste Wiersig-Wiechman for advice on histology and in vivo electrophysiology.

C. Linster was supported by National Science Foundation (NSF) Grant IBN-9723947. B. P. Wyble was supported by a predoctoral award from the NSF.

Present address and addres for reprint requests: C. Linster, Dept. of Psychology, Boston University, 64 Cumminton St., Boston, MA 02215. 
Received 27 July 1998; accepted in final form 10 February 1999.

\section{REFERENCES}

Barkai, E., Bergman, R. E., Horwitz, G., and Hasselmo, M. E. Modulation of associative memory function in a biophysical simulation of rat piriform cortex. J. Neurophysiol. 72: 659-677, 1994.

BARKaI, E. AND Hasselmo, M. E. Modulation of the input/output function of rat piriform cortex pyramidal cells, J. Neurophysiol. 72: 644-658, 1994.

Ben-Ari, Y., KRnJevic, K., Reinhardt, W., and Ropert, N. Intracellular observations on the disinhibitory action of acetylcholine in the hippocampus. Neuroscience 6: 2475-2484, 1981.

Biella, M. ANd De Curtis, M. Associative synaptic potentials in the piriform cortex of the isolated Guinea-Pig brain in vivo. Eur. J. Neurosci. 7: 54-64, 1993.

BILKeY, D. K. AND Goddard, G. V. Medial septal facilitation of hippocampal granule cell activity is mediated by inhibition of inhibitory interneurones. Brain Res. 30: 99-106, 1985.

Brashear, H. R., Zaborszky, L., AND Heimer, L. Distribution of GABAergic and cholinergic neurons in the rat diagonal band. Neuroscience 17: 439442, 1986.

Cauthron, J. L. and Stripling, J. S. Differential effects of urethane on potentials evoked in the olfactory bulb and piriform cortex by stimulation of the lateral olfactory tract and cortical association fibers. Soc. Neurosci. Abstr. 24: 256.3, 1998.

Elaagouby, A., Ravel, N., and Gervais, R. Cholinergic modulation of excitability in the rat olfactory bulb: effect of local application of cholinergic agents on evoked field potentials. Neuroscience 45: 653-662, 1991.

FANTIE, B. D. AND GodDaRd, G. Septal modulation of the population spike in the fascia dentata produced by perforant path stimulation in the rat. Brain Res. 9252: 227-237, 1982.

HABERLY, L. B. Summed potentials evoked in opossum prepiriform cortex. J. Neurophysiol. 36: 775-788, 1973.

HABERLY, L. B. Neuronal circuitry in the olfactory cortex: anatomy and functional implications. Chem. Senses 10: 219-238, 1985.

HABERLY, L. B. AND BOWER, J. M. Olfactory cortex: model circuit for study of associative memory. Trends Neurosci. 12: 258-264, 1989.

Hasselmo, M. E. Acetycholine and learning in a cortical associative memory. Neural Comp. 5: 32-44, 1993.

Hasselmo, M. E., ANDERson, B. P., ANd Bower, J. M. Cholinergic modulation of cortical associative memory function. J. Neurophysiol. 67: 1239-1246, 1992.

Hasselmo, M. E. AND Bower, J. M. Cholinergic suppression specific to intrinsic not afferent fiber synapses in rat piriform cortex. J. Neurophysiol. 67: 1222-1229, 1992.

Hasselmo, M. E. AND Bower, J. M. Acetycholine and memory. Trends Neurosci. 16: 218-222, 1993.

Hasselmo, M. E. And Linster, C. Modeling the piriform cortex. In: Cortical Models. Cerebral Cortex, edited by E. G. Jones, and P. S. Ulinski. New York: Plenum, 1998, vol. 13.

Hasselmo, M. E., LinSter, C., MA, D., AND CeKIC, M. Noradrenergic suppression of synaptic transmission enhances "signal-to-noise." J. Neurophysiol. 77: 3326-3339, 1997.

Hasselmo, M. E. And Schnell, E. Laminar selectivity of the cholinergic suppression of synaptic transmission in rat hippocampal region CA1: computational modeling and brain slice physiology. J. Neurosci. 14: 3898-3914, 1994.

Hunter, A. J. AND MurRay, T. K. Cholinergic mechanisms in a simple test of olfactory learning in the rat. Psychopharmacology 99: 270, 1989.

Ketchum, K. L. AND Haberly, L. B. Membrane currents evoked by afferent fiber stimulation in rat piriform cortex. I. Current source-density analysis. J. Neurophysiol. 69: 248-260, 1993.

KRNJEVIC, K. AND RoperT, N. Septo-hippocampal pathway modulates hippocampal activity by a cholinergic mechanism. Can. J. Physiol. Pharmacol. 59: 911-914, 1981

KRNJEVIC, K. AND ROPERT, N. Electrophysiological and pharmacological characteristics of facilitation of hippocampal population spikes by stimulation of the medial septum. Neuroscience 7: 2165-2183, 1982.

Kunze, W. A., Shafton, A. D., Kemm, R. E., and McKenzie, J. S. Effect of stimulating the nucleus of the horizontal limb of the diagonal band on single unit activity in the olfactory bulb. Neuroscience 40: 21-27, 1991.

Kunze, W. A., Shafton, A. D., Kemm, R. E., and McKenzie, J. S. Intracellular responses of olfactory bulb granule cells to stimulating the horizontal diagonal band nucleus. Neuroscience 48: 363-369, 1992.
LINSTER, C. AND GERVAIS, R. Investigation of the role of interneurons and their modulation by centrifugal fibers in a neural model of the olfactory bulb. J. Comp. Neurosci. 3: 225-246, 1996.

Linster, C. AND Hasselmo, M. Modulation of inhibition in a model of olfactory bulb reduces overlap in the neural representation of olfactory stimuli. Behav. Brain Res. 84: 117-127, 1997a.

LinSTER, C. AND Hasselmo, M. E. Olfactory delayed match-to-sample in a combined model of olfactory bulb and cortex. In: Computational Neuroscience, Trends in Research, edited by J. M. Bower. New York: Plenum, 1997b.

Luskin, M. B. AND PRICE, J. C. The distribution of axon collaterals from the olfactory bulb and the nucleus of the horizontal limb of the diagonal band to the olfactory cortex, demonstrated by double retrograde labeling techniques. J. Comp. Neurol. 209: 249-263, 1982.

Nickell, W. T. AND ShIPLEY, M. T. Neurophysiology of magnocellular forebrain inputs to the olfactory bulb in the rat: frequency potentiation of field potentials and inhibition of output neurons. J. Neurosci. 8: 4492, 1988.

Nickell, W. T. AND ShIPLEY, M. T. Evidence for presynaptic inhibition of the olfactory commissural pathway by cholinergic agonists and stimulation of the nucleus of the diagonal band. J. Neurosci. 13: 650-659, 1993.

Paolini, A. G. And McKenzie, J. S. Effects of lesions in the horizontal diagonal band nucleus on olfactory habituation in the rat. Neuroscience 57: 717-724, 1993.

Paolini, A. G. And McKenzie, J. S. Lesions in the magnocellular preoptic nucleus decrease olfactory investigation in rats. Behav. Brain Res. 81: 223-231, 1996.

Patil, M. M. And Hasselmo, M. E. Cholinergic modulation of synpatic inhibition in the piriform cortex. Soc. Neurosci. Abstr. 717: 9, 1996.

Paxino, G. And Watson, C. The Rat Brain in Stereotaxic Coordinates. San Diego, CA: Academic, 1986.

Perio, A., Terranova, J. P., Worms, P., Bluthe, R. M., Dantzer, R., and BIZIERE, K. Specific modulation of social memory in rats by cholinomimetic and nootropic drugs, by benzodiazepine inverse agonists, but not psychostimulants. Psychopharmacology 97: 262-268, 1989.

Ravel, N., AKaOKa, H., Gervais, R., AND Chouvet, G. The effect of acetylcholine on rat bulbar unit activity. Brain Res. Bull. 24: 1-5, 1990.

Ravel, N., Elaagouby, A., and Gervais, R. Scopolamine injection into the olfactory bulb impairs short-term olfactory memory. Behav. Neurosci. 108: 317-324, 1994.

Rovira, C., Ben-Ari, Y., AND Cherubini, E. Dual cholinergic modulation of hippocampal somatic and dendritic field potentials by the septo-hippocampal pathway. Exp. Brain. Res. 49: 151-155, 1983.

Rovira, C., BEn-Ari, Y., AND Cherubini, E. Somatic and dendritic actions of gamma-aminobutyric acid agonists and uptake blockers in the hippocampus in vivo. Neuroscience 12: 543-555, 1984.

Rovira, C., Cherubini, E., AND Ben-Ari, Y. Opposite actions of muscarinic and nicotinic agents on hippocampal dendritic negative fields recorded in rats. Neuropharmacology 21: 933-936, 1982.

SofFIE, M. AND LAMBERTY, Y. Scopolamine effects on juvenile conspecific recognition in rats: possible interactions with olfactory sensitivity. Behav. Processes 17: 181-199, 1988.

Tseng, G. F. AND Haberly, L. B. Deep neurons in piriform cortex. II. Membrane properties that underlie unusual synaptic responses. J. Neurophysiol. 62: 386-400, 1989.

Williams, S. H. AND Constanti, A. Quantitative effects of some muscarinic agonists on evoked surface-negative field potentials recorded from the guinea-pig olfactory cortex slice. Br. J. Pharmacol. 93: 846-854, 1988a.

Williams, S. H. AND Constanti, A. A quantitative study of the effects of some muscarinic antagonists on the guinea-pig olfactory cortex slice. $B r . J$. Pharmacol. 93: 855-862, 1988b.

Zaborsky, L., CARlSEN, J., BRAShear, H. R., AND Heimer, L. Cholinergic and GABAergic afferents to the olfactory bulb with special emphasis on the projections in the nucleus of the horizontal limb of the diagonal band. J. Comp. Neurol. 243: 488-509, 1986a.

Zaborszky, L., Heimer, L., Eckenstein, F., and Leranth, C. GABAergic input to cholinergic forebrain neurons: an ultrastructural study using retrograde tracing of HRP and double immunolabeling. J. Comp. Neurol. 250: 282-295, 1986b.

Zimmer, L. A, EnNis, M., AND Shipley, M. T. Diagonal band (NDB) stimulation increases the spontaneous activity of cells in the piriform cortex (PC) in vivo. Soc. Neurosci. Abstr. 22: 1031 (410.9), 1996. 